\title{
CRISIS ECONÓMICA, GASTO SANITARIO Y DESIGUALDADES EN SALUD. REFLEXIONES DESDE LA ECONOMÍA DE LA SALUD ${ }^{1}$
}

\author{
Autor: Guillem López i Casasnovas ${ }^{2}$ \\ CRES-Universitat Pompeu Fabra
}

\section{Resumen}

En las líneas que siguen se evalúa lo que sabemos y lo que desconocemos sobre el estudio de las desigualdades en salud como campo de investigación en la Economía de la Salud desde la perspectiva de las políticas públicas. Para ello el texto revisa el estudio del impacto de la crisis en la salud, los campos empíricos de validación, sus variables más determinantes asociables al ciclo económico, sus efectos en el capital social de los países y su causalidad reversa con los supuestos de crecimiento económico. Asimismo se comentan las principales medidas de amortiguamiento de la crisis en los factores sociales determinantes de la salud y las variables de intervención en políticas públicas

\footnotetext{
${ }^{1}$ Agradezco la ayuda a la preparación de este texto a Uxio Meis, investigador del CRES. Me he beneficiado por lo demás de los comentarios de Marc Sáez, Ignacio Abásolo, Laia Maynou y Hector Pifarré, aunque no son responsables de los errores, así como de los comentarios de dos evaluadores anónimos.

${ }^{2}$ guillem.lopez@upf.edu ORCID: 0000-0001-8020-9987.
} 
que intentan neutralizar mínimamente sus consecuencias. El texto concluye con una reflexión sobre aquellos aspectos del análisis que dificultan en mayor medida pasar del diagnóstico al pronóstico en la realidad de la implementación de las políticas públicas.

Palabras clave: crisis económica; desigualdades en salud; Economía de la Salud; políticas públicas.

\title{
Economic crisis, health spend and health inequalities. Reflections from health economics.
}

\begin{abstract}
The following lines analyze what we know and we do not know about the study of health inequalities as a field of research in Health Economics from the perspective of the existing public policies. For this purpose, the paper reviews the study of the impact of the crisis on health, the empirical fields of validation, the most determinant variables associated with the economic cycle, their effects on the social capital of countries and their reverse causality of its interrelation with economic growth. It also discusses the main measures of cushioning the crisis in those social determinants which affect health and the variables of intervention in public policies that try to neutralize their consequences minimally. The text concludes with a reflection on those aspects of analysis that complicate to move from diagnosis to prognosis in the implementation of public policies.
\end{abstract}

Key words: economic crisis; inequalities in health; health economics and public policies.

\section{INTRODUCCIÓN}

En julio de 2010 tuve la oportunidad de clausurar la Conferencia Europea de Economía de la Salud (ECHE 2010) celebrada en Helsinki con la ponencia plenaria y en el momento pionera "Los ciclos económicos, la salud y el cuidado de la salud", como presidente en aquel momento de la Asociación Internacional de Economía de la Salud (IHEA). Desde entonces han sido múltiples las 
aportaciones al tema ${ }^{3}$. En las líneas que siguen evalúo lo aprendido y reflexiono en general sobre el estudio de las desigualdades en salud como campo de investigación en la Economía de la Salud desde la perspectiva de las políticas públicas asociables a las reflexiones anteriores. Para ello reviso el estudio del impacto de la crisis en la salud, los campos empíricos de validación, sus variables más determinantes resultantes del ciclo económico, sus efectos en el capital social de los países y su causalidad reversa con los supuestos de crecimiento económico, sus principales medidas de amortiguamiento y las variables que aseguran neutralizar mínimamente sus consecuencias. El texto concluye con aspectos que dificultan pasar del diagnóstico al pronóstico en la realidad de las políticas públicas.

\section{EL IMPACTO DE LA CRISIS EN LA SALUD}

En el dominio de determinantes de la salud, el 70\% de los factores son componentes no-genéticos, siendo las dos terceras partes de estos factores sociales y de comportamiento: esta es la parte más arriesgada a la hora de valorar el impacto de la crisis económica en la salud. El tercio restante es el cuidado de la salud.

Es sabido que el desempleo está vinculado a la mala salud y se ha asociado a un aumento de las tasas de mortalidad, especialmente de las enfermedades del corazón y el suicidio (Jagger et al., 2008). Cierta evidencia opuesta del otro lado del atlántico, en la mejora de ejercicio y estilo de vida cuando el individuo se queda sin trabajo, pero de extrapolación dudosa a otras situaciones.

En general, para combatir la ansiedad y el aislamiento social de las pérdidas de empleo, la pobreza, el paro erosionante del capital humano, la peor nutrición y el comportamiento poco saludable devienen temas clave.

Dada la desigualdad en rentas creada por la situación laboral, el ajuste económico podrá incidir de modo desigual contando para ello menos las políticas de welfare más universalistas que las económicas selectivas de workfare ${ }^{4}$.

En cualquier caso, son parte de un nuevo enfoque de la epidemiología y su relación con los factores determinantes macrosociales de la salud, aspectos tales como: (i) La equidad entre generaciones (el bienestar de los niños: el capital humano del futuro); (ii) las prácticas fiscales -que en sistemas fiscales duales conducen a la regresividad-; (iii) la brecha educativa y sus efectos sobre la desigualdad por la vía intermedia del tabaquismo y el sobrepeso; (iv) regulaciones urbanísticas y medioambientales (zoning con incidencia en la movilidad y finalmente en

\footnotetext{
${ }^{3}$ Literatura recientemente revisada por Parmar, Stavropoulou y Ioannidis (2016).

${ }^{4}$ Una discusión sobre ello se puede encontrar en Lundberg, O. et al. (2008).
} 
enfermedades respiratorias y obesidad); (v) prácticas empresariales (producción y diseño, la comercialización y la distribución al por menor y precios) en sus efectos sobre el consumo y hábitos de nutrición.

Adicional y superpuestas a lo anterior, cuenta y mucho la evolución de la estructura económica, de los mercados de trabajo y los modelos de bienestar (las políticas familiares en general, o más orientadas al mercado) y la incidencia que en todo ello pueda tener la migración.

\section{Los campos de validación}

1. Los factores de interrelación de las variables explicativas convencionales: ingresos, pobreza, desigualdad, educación, estilos de vida. Existen datos para países europeos de la OCDE (Disparidades en el estado de salud y el acceso a los servicios sanitarios. OCDE WP de marzo de 2009). De este estudio podemos derivar:

- En el ingreso: Grecia y Alemania son los países que dependen en mayor medida de las fluctuaciones, vista la elasticidad de ingresos para la cobertura de salud

- Sobre la desigualdad asociada a la renta: Bélgica, Reino Unido e Irlanda se sitúan en las cotas peores dadas las relaciones observadas entre sus deciles de renta.

- Sobre la pobreza europea: la exposición mayor (por tasas elevadas en gasto sanitario más catastrófico) la tienen España, Portugal y Suiza.

- En los estilos de vida: el acceso deseado y no satisfecho en necesidad de examen médico, se mostraba superior en Polonia, Portugal, Italia, Grecia y Eslovaquia.

- En las diferencias sociales en educación los peores coeficientes los tenían Polonia, Hungría, Países Bajos y República Checa.

Pero, en términos globales, no existe suficiente evidencia convincente sobre las relaciones observadas en el pasado con el fin de predecir el futuro. Se necesitan mejores métodos (de tipo de diferencia en diferencia) dentro del enfoque de datos de panel individual micro.

2. Estudio de mecanismos: no está claro en los estudios las vías de transmisión o mecanismos con los que operan los cambios postulables; así en espacios de tiempo breves sobre la renta, el empleo, etc. y cómo ellos pueden afectar las tendencias básicas más estructurales: de la inversión de capital humano, el comportamiento de los individuos o en el consumo en el ciclo de vida del ingreso permanente. 
3. En cualquier caso, no resulta obvio de que incluso superando lo observado como dificultades analíticas de (1) y (2) las políticas actuales de salud pública puedan cambiar la dirección de las tendencias.

Se necesita para ello, a nuestro entender, (i) más análisis de la Epidemiología y la Macroeconomía de los factores sociales, (ii) mejorando la modelación, por ejemplo de las adicciones racionales que posponen reacciones y (iii) un enfoque empírico que mediante la instrumentación de las variables adecuadas neutralice la endogeneidad, y así requiriendo políticas específicas para los diferentes grupos afectados. Por ejemplo, vista la existencia de causalidad acumulativa en los procesos de acondicionamiento -social, laboral tecnológico a las nuevas circunstancias de la relación capital- trabajo, desenmarañando el sesgo de muestreo -debido en su caso a la heterogeneidad por aspectos individuales no observables en las correlaciones como entre el hábito de beber y obesidad con fumar, que afecta a su vez a la productividad y a los salarios. Asimismo cabría reformular algunos aspectos relativos a la política fiscal de tributación a consumos nocivos habida cuenta la regresividad del impuesto; a las políticas alimentarias (lo más sano más subsidiado o lo más nocivo con extra coste fiscal). Todo ello desde una óptica de reconocimiento de fuertes interrelaciones; así en el consumo medio de alcohol y su dependencia del ciclo económico y, por tanto, con acciones universales para prevenir el abuso de alcohol durante la crisis de mayor dificultad para que no sean infructuosas. De manera similar para los aumentos de desempleo más o menos compensables en la ingesta de alcohol condicional a la renta, y así requiriendo políticas específicas para los diferentes grupos afectados (Todeschini, 2010).

Sin embargo, más allá de la evidencia más o menos contrastada, el sentido común dice que:

(I) Los factores de riesgo para la salud asociables a la crisis económica son: el desempleo, la mayor ansiedad, la alimentación menos sana y la acumulación de menor capital humano.

(II) Los efectos de la restricción presupuestaria inciden de modo diferente en el gasto social. Así, el consumo público para la provisión de servicios deviene más protegido que la financiación de las transferencias sociales. Los gastos de atención sanitaria están en riesgo menor en un mundo keynesiano en el que se consideren los multiplicadores del gasto. Más dudoso es sin embargo el impacto de la crisis financiera sobre las políticas que inciden en los determinantes sociales y económicos.

(III) Los efectos finales dependen previsiblemente mucho más de la familia y el modelo básico de asistencia social prevalente, la cuantía de los activos de riesgo compartido y la composición del porfolio de activos financieros 
más o menos expuestos a la volatilidad financiera; perceptores duales en ingresos de familia, etc.

(IV) Necesitamos para ello más que nunca ser selectivos con las políticas a implementar, a medida para grupos beneficiarios y más frágiles en particular. La edad no parece para ello un factor determinante. La varianza y no la media es el elemento relevante en la definición de dicho nuevo tipo de políticas públicas.

\section{LAS INTERRELACIONES ENTRE ECONOMÍA Y SALUD EN EL CICLO ECONÓMICO}

En la medida en que continúa la crisis económica, son muchas las voces, aunque menos los análisis, que alertan de la concatenación que se estaría produciendo entre pérdida de renta, desigualdades socioeconómicas y su impacto sobre la salud (López-Casasnovas, 2012). Tiene por ello sentido una reflexión sobre las cuestiones vinculadas a dicha secuencia ${ }^{5}$. El mensaje principal de los estudios revisados es (i) que la evidencia de la relación entre crisis y desigualdades en salud es por el momento elusiva, aunque probable, y que los mecanismos de salvaguarda de daños de la crisis sobre la salud no tiene contra-factuales ajustados claros para la acción de la política sanitaria, y (ii) que en cualquier caso nuestro sistema de salud, anquilosado en sus inercias, no parece responder hoy con cambios en sus dispositivos, orientados a enfrentarse a la parte del problema que se pueda considerar prevenible (que no es toda).

\subsection{Una secuencia de concatenación elusiva}

La literatura disponible acerca de cómo las crisis económicas afecta la salud de las poblaciones es dicotómica. Algunos autores argumentan los efectos de la crisis en positivo (experiencias en EE. UU. básicamente) y otros en negativo (caso de la descomposición de las repúblicas soviéticas) y, entremedio, las que detectan un factor negativo neutralizado por las redes del Estado del Bienestar (la crisis de Finlandia como ejemplo). De todo ello hay un número prolijo de aportaciones. De la supuesta experiencia en EE. UU., se cuenta que la crisis y el aumento del paro reducen el estrés poblacional, disminuye los costes de oportunidad de algunos comportamientos saludables (ejercicio, control dietético) y aumenta los de los insanos (tabaco y alcohol ante una disminución de la renta). De todas estas hipótesis son posibles, sin embargo, concatenaciones inversas, por lo que la parte

${ }^{5}$ Lo hago desde el poso propio de conocimiento de la literatura y de la evidencia que he podido derivar de mi participación durante dos años como senior advisor en la Comisión de la OMS sobre Desigualdades en Salud (conocida también como Comisión Marmott). 
empírica es decisiva. Del caso soviético, son precisamente los consumos nocivos los que se asocian a la crisis, con efectos graves sobre la morbi-mortalidad de la población. Pero la sospecha de que podría haber habido un cambio en las estadísticas que se hayan dejado de manipular con la Perestroika, puede ofrecer también una explicación plausible. En todo caso, tampoco parece razonable considerar, como resultado de la evidencia soviética, que la situación dictatorial anterior era 'mejor' habida cuenta los efectos que la apertura de la economía y posterior crisis económica ha tenido para la salud poblacional. Además, los factores explicativos podrían no deberse tanto a la desigualdad de la renta (cuestión más fácilmente cuantificable) como a la corrupción y a la ausencia de capital social, intrínsecos a tantos años de regímenes comunistas autoritarios. Ya sabemos que, en general, cuanto más libre es una economía, más desigualdad parece conjugada al mayor crecimiento; cuanto más abiertas las fronteras a la inmigración, más desigualdad social, aunque para los inmigrantes, el 'voto con los pies' es síntoma inequívoco de su propia mejora. Y a sensu contrario, economías cerradas y con telón de acero hacen sociedades más iguales, más pobremente iguales. Finalmente, el análisis finlandés (y en menor grado el sueco de los noventa) no tiene contrafactual fácil en tiempo y lugar: la crisis no tuvo incidencia en la salud de la población, pero la contribución del Estado del Bienestar no permite obviamente ser valorado respecto de lo que hubiera acontecido en su ausencia. Un experimento que ciertamente nadie creo estaría interesado en sufrir en propias carnes.

Por lo demás, las valoraciones empíricas tienen una validez limitada; limitada (i) al período observado, sin clara capacidad extrapolativa; (ii) al país en cuestión, su cultura, la idiosincrasia de su gente, la naturaleza del sistema de protección social, o a los efectos composición que van de lo individual a lo comunitario; (iii) al funcionamiento efectivo del dispositivo asistencial y de sus instituciones en la práctica: así en contextos de flexi-seguridad o de mercados de trabajo rígidos; a igual cifra de desempleo, en la combinación de parados de larga duración (desánimo y cronicidad acelerada en el tiempo) que de jóvenes "ni-nis" (con efectos previsiblemente a más largo plazo); a igual stock de parados, entre entrantes y salientes por grupos de edad (es sabido que para los mayores, la incidencia marginal de las desigualdades económicas respecto de las desigualdades en salud dentro de su cohorte es menor que en el grupo de jóvenes); según el acompañamiento de política social que proceda, ya sea pasiva (welfare), o activa -vía mercado de trabajo (workfare), etc. Esta es una observación general para distintos análisis de política sanitaria que no permite extrapolación sencilla: así cuando algunos predicen los efectos de limitar la utilización de algunos servicios supuestamente para contener costes como consecuencia de la crisis de las finanzas públicas. Para ello, algunos utilizan resultados de otros países, por ejemplo en elasticidades precio ante copagos, haciendo caso omiso de la etapa analizada, del país en cuestión, de su dispositivo asistencial (alternativas asistenciales complementarias y substituti- 
vas), sus interdependencias y filtros (copagos en un ámbito y no en otro); según tipo de copagos (un fijo, un porcentaje, con o sin deducible, con o sin techos), establecido donde no lo había o aumentado donde ya existía, sobre la base de un mayor desembolso o de un menor reembolso, vinculado o no a la renta, etc.

¿Qué sentido tiene pues utilizar elasticidades del proyecto de la RAND para los setenta en EE. UU. a conveniencia (son múltiples y distintos sus resultados por colectivos en tipos de utilización y con distintos grados de efectividad) para aplicarlos para el caso español? ¿Qué lógica tiene considerar quintiles de renta para medir la desigualdad en su incidencia sobre la salud desde una perspectiva temporal por parte de aquellos mismos que critican la renta como medida del bienestar, ignorando así el acceso de todas las decilas a mayores y mejores bienes de capital y beneficios en especie públicos y privados, colaterales a la consecución de renta en un momento dado? ¿Qué esfuerzo se observa en el análisis de aquellos estudiosos para neutralizar las diferencias de salud que se derivan del simple cambio demográfico? Nuestros mayores, aún con una desigualdad socioeconómica más elevada, al acumularse en el tiempo, son "más iguales" en su peor salud que los jóvenes; sanos en su stock inicial, sean las que sean sus diferencias socioeconómicas. ¿O por diferencias en morbi-mortalidad por acceso a tecnologías tan dispares como socialmente aceptadas (cuidado de prematuros, de terminales) o de comportamientos que priorizan en el bienestar individual, si responsablemente, consumos nocivos (más allá de un cierto paternalismo en libertad "cada uno se suicida como quiere”, con tabaco, exceso de ingesta calórica, etc.)? ¿Qué parte de no corrección de aquella morbi-mortalidad, más allá de la educación para la salud, es imputable a un sistema sanitario o de protección social que se pueda considerar más efectivo? En resumen, la concatenación entre crisis económica, desigualdades en renta y en salud no tiene bases empíricas robustas, a menudo ni en las estimaciones econométricas originarias, y en muy pocos casos permite extrapolaciones de resultados fuera de tiempo y lugar. Es por ello campo abonado a pre-juicios políticos en el que muchos analistas de política sanitaria imponen conjeturas y predicciones.

\subsection{Del diagnóstico a la prognosis}

Aún pese a tan escasa evidencia que sea replicable urbi et orbe, dado su carácter más elusivo en causalidades que en su más que probable correlación, conviene interrogarnos de cuál es, o puede ser, la respuesta de los dispositivos asistenciales a los problemas que se puedan estar larvando y así detectar con la crisis y su permanencia. Ciertamente los efectos si se producen, pueden visualizarse más en el largo que en el corto plazo, no ignorando que suicidios y depresiones agudas pueden jugar su papel en el corto plazo y con mucha dureza. Sin embargo, a nivel agregado la crisis puede mostrar su peor cara en la aceleración de procesos de cronicidad, no tanto en el momento de paro o de pérdida de renta como en la de consumo, desahorro o 
pérdida patrimonial; no en el paro sino en la pérdida del subsidio, y vista la duración del desempleo, ya con o sin red familiar, con o sin cargas o responsabilidades frente a terceros. Aceptando, por tanto, que la crisis trastoca el statu quo familiar y personal de los ciudadanos, emergiendo problemas actuales de salud o encubriendo nuevos problemas a futuro, la pregunta relevante es la de si los sistemas de protección social están teniendo la orientación y respuestas apropiadas a las nuevas situaciones. Frente a ello, un sistema que algunos analistas valoran por su consolidación es a menudo un sistema fácilmente anquilosado que se mueve por inercias. Un sistema de protección social fragmentado, en el que importa más quién y en qué se gasta, más que para qué y cómo se gasta, no parece ser adecuado para afrontar nuevos retos. Acabar con los silos departamentales de nuestra administración obligaría a una mayor coordinación entre las políticas de welfare (convenidas al "no trabajo") con las de workfare (precisamente condicionando la ayuda al trabajo). La prevención de las situaciones por las que los condicionantes socioeconómicos pueden impactar en la salud se debiera de buscar en la ubicación del nicho que causa el problema: si el paro, en los condicionamientos al recibir las ayudas, o en los propios cursos de formación de parados. ¿Existen módulos acaso de naturaleza sanitaria o de protección de la salud en dicha formación? ¿Se explica al parado, la relevancia de las comparativas? Caminando, sin costes de oportunidad elevados respecto de otras formas de transporte público, en cuanto a diferenciales de tiempo -no de tiempos totales-, de costes monetarios y de beneficios en salud derivados del simple hecho de andar. ¿Se expresa la prevención mayormente en los crónicos que en las poblaciones asintomáticas? ¿Responde la oferta asistencial haciendo más de lo mismo o cambiando la composición de lo que se hace? ¿Existe efectivamente con la crisis más atención a la salud mental del parado de larga duración y menos a revisiones de poblaciones sanas? ¿Se educa al parado para que sepa sacar máximo provecho de su renta, a través de consumos más austeros, más ricos en nutrientes e igualmente complacientes? Así, en el valor nutritivo de algunos desechos por hábito de no consumo (la piel de una patata limpia, insumos de temporada, la fruta en caducidad) y de aprovechamientos alimenticios conocidos de etapas más frugales. Nada apunta a que ello sea objeto de preocupación de nuestras políticas, más centradas en la defensa del estado de cosas que en su evaluación o experimentación de nuevas estrategias. Los argumentos asociados a la amenaza de la crisis sobre la salud se utilizan a menudo como punto de escape eludir la consolidación fiscal.

\section{DESIGUALDADES EN SALUD, SOCIEDADES INCLUSIVAS Y CRE. CIMIENTO ECONÓMICO}

La otra cara del análisis tiene que ver con los beneficios de reducir las desigualdades en salud, precisamente como impulso al crecimiento económico para 
la salida de la crisis. El crecimiento inclusivo, con una creación de riqueza menos desigual, con los ingresos mejor distribuidos como fórmula para generar más salud con suficiente equidad sanitaria para acompañar el crecimiento económico con un mayor desarrollo social. Sin embargo, para poner a prueba esta secuencia positiva, con todos los vínculos asumidos, su validación demuestra ser muy difícil. Esto tiene que ver, por un lado, con la cuestión de la causalidad inversa (en la identificación de qué causa qué) y por otro lado con la heterogeneidad de la muestra y la diversidad observada en las comparativas y con múltiples limitaciones en los datos.

La literatura sobre el crecimiento económico y la salud es extensa. Los determinantes socioeconómicos de la salud proporcionan el vínculo oculto para los estudios (OMS, 2013). Sin embargo, el análisis del impacto del crecimiento económico sobre la salud, y el impacto de la salud en el crecimiento económico, sigue siendo una interrelación muy compleja para la investigación en economía de la salud (López-Casasnovas y col., 2005). Muchas de las contribuciones intentan modelar los efectos de salud en el crecimiento económico, mientras que otras se centran en su reverso (Bhargava et al., 2001): cómo cambia la salud como resultado del crecimiento económico (López-Casasnovas y Soley-Bori, 2014). Aunque existen algunos problemas de endogeneidad en ambos enfoques, particularmente en el crecimiento económico tomada como variable, ya que su variación puede explicarse, entre otras razones, el nivel de salud de una población y en particular sobre las desigualdades en su distribución.

En general, estos enlaces relevantes pueden ser probados más fácilmente a nivel macro, a través de la dinámica de la salud y de los ingresos, en un estudio de corte transversal (para un país específico) o en el nivel micro probando si los más pobres hoy en día, en un lugar de altos ingresos y desigualdad, disfrutan de mejor salud que en el pasado. Además, es probable que sea no lineal la relación entre el ingreso y la salud y puede ser afectada diferencialmente por los impactos a corto plazo. Por otro lado, la perspectiva estándar es que las desigualdades en salud parecen referirse principalmente a la pobreza y no tanto a la desigualdad de renta (Leigh et al., 2009). Por lo tanto, teniendo en cuenta los patrones dinásticos de la salud y la pobreza, más atención debería dedicarse a la dinámica de la salud a lo largo del ciclo de vida de los individuos y de los mecanismos de transmisión entre generaciones, y no tanto a las diferencias de los países de muy diversas experiencias.

En la vida real, existen desigualdades en salud entre y dentro de los países. La Comisión sobre Macroeconomía y Salud señaló ya en su día que podría haber un costo considerable en términos de crecimiento económico no percibido como resultado de que algunos países mantengan un nivel mucho más bajo de la salud en general que en otros. Algunas investigaciones han documentado los beneficios económicos significativos en forma de un crecimiento económico más rápido 
gracias a la reducción de las desigualdades en salud entre países. La evidencia (Suhrcke y Cookson, 20126) proviene de países de ingresos altos de la OCDE, así como de los países de Europa y Asia central y oriental. Sin embargo, sigue existiendo un debate científico sobre el grado en que el nexo entre la salud y el crecimiento realmente refleja una relación causal. En este sentido, tal como se desprende de una extensa investigación, el efecto económico adverso de la mala salud se analizó mejor que la desigualdad en la salud y de modo mucho menos controvertido que a nivel individual. Como entre los grupos socioeconómicos son los inferiores los que sufren peores condiciones de salud y por lo tanto deben incurrir en mayores pérdidas económicas (en forma de pérdida de ingresos y acceso al mercado de trabajo), sobre dicha base se argumenta que las desigualdades de salud es probable que impongan una carga económica considerable para la sociedad. La verdadera carga económica de las desigualdades en salud, sin embargo, se encontraría en el valor que las personas atribuyen a las vidas perdidas y a la calidad con la que se viven como resultado de las diferencias socioeconómicas. Ese valor es difícil de medir, pero sin duda supera con creces cualquier beneficio estrechamente considerado, y posiblemente inexistente en las cuentas de resultados en forma de ingresos adicionales o ahorros en los costos de atención médica, que es poco probable que resulten de dichas políticas.

Como Suhrcke y Cookson (2012) han señalado acertadamente, los estudios de carga económica de los costos de la desigualdad en salud sin duda puede ayudar a llevar el tema a la atención de los responsables de las políticas fuera del sector de la salud. Sin embargo, estudios de carga económica no pueden apoyar el caso a favor o en contra de las acciones específicas de política. De modo que estudios que se centran en estimar las cargas financieras son un arma de doble filo que puede distraer la atención de otras cuestiones más importantes para la salud y el bienestar humanos. En consecuencia, aquellos que tratan de avalar acciones políticas específicas para hacer frente a las desigualdades en salud deben considerar el uso de los repositorios públicos existentes de estudios de evaluación económica creíbles (CEA, análisis coste-efectividad) y CBA (análisis coste-beneficio) para hacer frente a posibles reclamaciones exageradas que estas políticas puedan tener de perjudiciales. En la búsqueda de evidencia, necesitamos, por ejemplo, CBAs acerca de las políticas públicas que operando fuera del sector de la salud que pueden influir en los gradientes sociales en la salud, en Europa específicamente en lugar de los Estados Unidos.

La evidencia ya disponible se basa en dos estudios que analizaron una muestra de 22 países desarrollados entre 1960 y 1985 . Sus autores encontraron que la salud -medida por la esperanza de vida- no tuvo un impacto significativo sobre el

\footnotetext{
${ }^{6}$ En opinión emitida acerca de los Determinantes sociales y la brecha de la Salud en la Región Europea de la OMS: El Informe final es de Marmott (2013).
} 
crecimiento económico o en los niveles de ingreso per cápita (Knowles y Owen 1995, 1997). ¿Quiere esto decir que, por encima de un cierto nivel de desarrollo económico, la continuación de las mejoras de salud o bien pueden no tener ningún impacto o incluso reducir el crecimiento económico posterior? No hay una respuesta definitiva a esta pregunta. Otras investigaciones recientes (Suhrcke y Urban, 2010), teniendo un cuidado considerable para superar el problema de endogeneidad utilizando un enfoque de panel dinámico, encuentran un muy robusto impacto "causal" de tasas de crecimiento per cápita en la esperanza de vida en una muestra de 26 países de rentas altas durante el periodo 1960-2000. En una estimación representativa de un ámbito asistencial concreto, una reducción del $10 \%$ en la mortalidad cardiovascular se asoció con un incremento de un punto porcentual en el crecimiento de la renta per cápita, una cantidad aparentemente pequeña pero con un gran efecto cuando se considera en el largo plazo. Sin embargo, el debate sobre el verdadero impacto de la salud en el crecimiento económico está lejos de ser resuelto.

Tras Suhrcke y Cookson (2012), pocos estudios han llevado a cabo una valoración económica completa de los costes de las desigualdades en salud (esto es, entre otros factores, debido al hecho de que no está claro cuál es la situación hipotética apropiada en las estimaciones). Dos estudios relevantes en este campo son Mackenbach et al. (2011) en los países de la UE-25 y Dow y Schoeni (2008) en los EE. UU. Mackenbach et al. (2004) perseguían dos enfoques diferentes en la medición de los costos económicos de las desigualdades en salud. Para la UE25 países, en su conjunto, las estimaciones de las desigualdades relacionadas con las pérdidas para la salud como un "bien de capital" (que conduce a menos mano de obra y a mayor productividad) ofrecen cifras modestas en términos relativos (1,4\% del PIB) pero elevadas en términos absolutos (141 mil millones de euros del 2004). También valoraron la salud como "bien de consumo" - que consiste en la aplicación del valor estadístico de la vida (VSL). Desde esta perspectiva más amplia, el impacto económico de las desigualdades socioeconómicas en la salud puede muy bien ser grande: en el orden de alrededor de 1.000 millones de euros, o el 9,5\% del PIB. Un tercer estudio se ha llevado a cabo para la revisión de la Comisión Marmott para Inglaterra (Mazzuco, Meggiolaro y Suhrcke, 2010). Este estudio también utilizó el VSL para convertir las desigualdades en salud en valores monetarios. En el escenario preferido por los autores, es decir, suponiendo que sólo una parte del gradiente de la mortalidad se reduce, encuentran que para la población adulta considerada en su conjunto, las ganancias económicas serían en promedio entre aproximadamente 98 y 118 mil millones de libras (a precios de 2002) en Inglaterra y Gales. Al dejar partes de la población sin contabilizar e ignorar las condiciones o enfermedades no mortales, las estimaciones es muy probable que representen el límite inferior de los verdaderos beneficios que podrían resultar de dichas reducciones. 
En general, los ingresos aumentan con la mejora de la salud, y la mejora de la salud sirve para múltiples fines distintos al del simple crecimiento de renta (Deaton, 2003). Sin embargo, el logro de este círculo virtuoso, no implica que el crecimiento siempre vaya acompañado de una mejora en la redistribución del ingreso, ni esto es una garantía para una mayor equidad en salud. El análisis del efecto de descomposición de la interrelación entre el ingreso y la salud va más allá de los vínculos individuales y se centra en la distribución conjunta de la salud y renta. En este menester surgen tres preguntas principales: (i) ¿cómo opera un aumento de la renta en su impacto en la salud a partir de diferentes estados de salud? $\mathrm{O}$, más precisamente, ¿cómo se traduce en el bienestar conjunto el incremento mayor de renta, especialmente los que están en peores condiciones de salud?; (ii) ¿es la función de bienestar cóncava en relación con la renta? Si es así, esto implicaría que por la simple transferencia de ingresos de ricos a los individuos más pobres, el bienestar social mejoraría; (iii) ¿supone la concavidad de la renta una disminución del bienestar social si acrece a quienes disfrutan ya de niveles de salud más altos y, como consecuencia, con el fin de mejorar el bienestar social una transferencia destinada a las personas pobres y enfermas tiene un impacto mayor que una dirigida a aquellos que son pobres pero cuentan con un buen estado de salud?

Con el fin de probar estas hipótesis, conviene observar algunas diferencias de medición entre la salud y la renta. Respecto de la primera, se utilizan comúnmente indicadores de salud, que como las evaluaciones de percepción de salud, no son cardinales, mientras que el ingreso sí lo es. En segundo lugar, hay límites superiores para la salud, pero no para la renta. En tercer lugar, quizás a excepción del caso de una donación de órganos, se puede transferir ingresos de una persona a otra, pero no se puede transferir la salud. Por último, los stock de salud iniciales, las diferentes capacidades para transformar recursos en salud y hasta qué punto todo el mundo consigue su condición óptima en particular (la de su máxima salud posible), requiere una estrategia bastante completa para compensar las diferencias de salud percibidas. Como resultado de estas particularidades, la heterogeneidad de la composición del estado de salud observado deviene esencial, ya que cuando se comparan dos distribuciones, con el fin de mostrar el "dominio" (entre países o grupos de individuos, por ejemplo), aparte del punto central, los intervalos de confianza se deben revisar. Cuanto más las distribuciones se solapan, más ambiguos los resultados del análisis serán, por lo que las decisiones de política resultarán mucho más difíciles de argumentar (López-Casasnovas y Soley-Bori, 2014).

También vale la pena señalar que la desigualdad de salud asociada con la renta está relacionada con el ingreso medio a través de la elasticidad-ingreso. Esta mide qué significa para la salud un aumento proporcional en el ingreso y cómo esta elasticidad ingreso varía con el nivel de ingresos a la vista de cómo aquella responde. Por otra parte, las desigualdades en salud relacionadas con los ingresos se ven influenciadas por factores distintos de la desigualdad de ingresos estrictamente 
hablando. Para ello, el efecto de la renta media en la consideración de la elasticidad necesita ajustarse por factores distintos a la renta y a su rango. Para las desigualdades relacionadas con los ingresos en sus aspectos supuestamente determinantes para la salud, tales como la edad, por ejemplo, y la forma en que se concentra ésta en los grupos sociales, aparecen como los factores más relevantes. En este sentido, Contoyannis y Forster (1999) muestran que una política pública que reduce la desigualdad de renta puede, bajo ciertas circunstancias, y teniendo en cuenta el signo de los anteriores efectos, dejar sin cambios la desigualdad en salud, o incluso aumentarla. Como tal, la elasticidad renta de la desigualdad en salud puede desempeñar un papel crucial ya que si aumenta con los ingresos, un incremento proporcional de la renta puede dar lugar a una mayor desigualdad en salud relacionado con los ingresos. De lo contrario, si el crecimiento de los ingresos va de la mano con una reducción de la desigualdad en salud, una mayor inclusión social derivaría como un beneficio añadido o externalidad positiva.

Con el fin sin embargo de justificar una política basada en la redistribución del ingreso mediante la reducción de los niveles promedio absolutos, sería necesaria una función muy específica (e inverosímil) de bienestar que identificase a aquellos que son "igualmente pobres, todos con los mismos niveles de salud", como una situación de mejora del bienestar. Por esta razón, algunos autores, entre ellos Fleurbaey y Schokkaert (2009), defensores de la consideración de las desigualdades en salud en el ámbito del bienestar en general, abogan por tener en cuenta las desigualdades en campos distintos a los socioeconómicos con el fin de reducir al mínimo las desigualdades "inaceptables" en el bienestar. Las desigualdades en estos otros ámbitos se deberían tener en cuenta para que la brecha de equidad existente sea entendida como la distancia entre la distribución real observada y una "distribución equitativa" en la que todos los efectos de las variables que dependen de la propia responsabilidad de los individuos, o los que se consideran inevitables, se eliminasen. Esto implica la necesidad de ajustar por aquellas diferencias entre los grupos debido a los gradientes de clases sociales exógenos, pero no dentro de los grupos de la misma clase "a la Roemer" (Roemer, 1998). Ello resultaría similar a una estandarización convencional mediante el ajuste de la regresión estimada para todas las variables por encima de un valor de referencia dado.

Debido a que uno podría estar dispuesto a sacrificar la salud con el fin de ganar más renta, y viceversa, la correlación entre el ingreso y la salud sobre una base individual puede ser valorada de modo diverso a pesar de que, a posteriori y a nivel agregado, más ingresos por lo general significan una mejor salud. Para entender esta relación, la posición exacta de renta del individuo es crucial. Si pertenece a una clase de mayores ingresos y la salud media mediante la reducción de la desigualdad de ingresos mejora, pero sin ningún efecto sobre la salud de la clase más baja de ingresos, la desigualdad de la salud relacionada con la renta aumentaría. Básicamente, para este resultado, tres factores principales influyen en los 
resultados de salud: la dotación inicial de la salud, la eficiencia en la producción de la salud, y cómo rendimientos decrecientes a escala es probable que aparezcan como el individuo se acerca a su óptima. En efecto, mediante la redistribución de los ingresos, las desigualdades pueden reducirse, así como la salud media de la población, pero esto dependerá en última instancia la reacción de los "productores de salud de bajos ingresos" a cualquier mejora en su renta.

Como consecuencia de todo ello, una vez que capturamos la configuración real de los enlaces bidireccionales (véase Rodríguez y González, 2011 vs. Mackenbach, 2011), la lucha contra las desigualdades en salud relacionadas con los ingresos requiere estrategias más complejas.

\section{En búsqueda de la prueba empírica}

Van Ourti et al. (2009) proporcionan algunas evidencias de los efectos del aumento de los ingresos y su distribución en la desigualdad de la salud en Europa. De hecho, analizan cómo varía la distribución del ingreso y la desigualdad de la salud relacionada con los ingresos y cómo desenmarañar el efecto del crecimiento de la renta en relación a los cambios en la desigualdad de ingresos y ésta en la salud y en su distribución. Ello se aproxima con la estimación de dos niveles de salud hipotéticos: en primer lugar, el nivel de salud que prevalecería en el caso de la distribución del ingreso no cambiase; y en segundo lugar, el nivel de salud que prevalecería en caso de un aumento de los ingresos proporcional (el puro componente de elasticidad renta). Esto permite aislar el efecto de los cambios en la distribución del ingreso de los otros determinantes de la salud, así como las variaciones de la desigualdad de ingresos ante un crecimiento de la renta proporcional. En ambos casos, hay un efecto directo de la redistribución del ingreso en las desigualdades de salud relacionadas con la renta, pero también un impacto indirecto, a través de los otros determinantes de la salud. La evidencia sugiere que los cambios favorables a los pobres en la desigualdad de ingresos no siempre conducen a reducciones en las desigualdades de salud relacionadas con la renta si la desigualdad del ingreso y la elasticidad no se mueven juntas "en promedio". Kunst y Mackenbach (1994) discuten el caso en el que los problemas de salud, que surgen inevitablemente en el transcurso del tiempo, actúan como niveladores y, como resultado, disparidades socioeconómicas tienden a reducirse a edades avanzadas. En particular, Deaton y Paxson (1998) encontraron que la salud se deteriora con la edad a un ritmo persistente, constante, y que la varianza de la salud aumenta hasta la edad de 60, después de lo cual permanece constante. Además, argumentan que si asumimos que los choques son acumulativos y no al azar, la predicción de una varianza creciente con la edad no se cumpliría. Estos autores también encontraron que el gradiente de la renta sobre la salud es mayor en las cohortes más jóvenes y que los componentes socioeconómicos de la desigualdad 
en la salud han ido en aumento, mientras que la desigualdad total en salud, medida por la varianza, ha estado cayendo.

En general, la mayor parte de la teoría aún tiene dificultades para encontrar evidencia apropiada sobre la causalidad de los efectos. La cuestión es identificar si es una mejor salud lo que impulsa un mayor crecimiento económico o todo lo contrario, esto es, el hecho de haber logrado un mayor desarrollo lo que conduce a una preocupación por el logro de niveles de salud más altos (en el sentido de valorar la salud como una especie de bien de lujo cuando otras necesidades básicas están cubiertas).

Los resultados de los asociación en términos de niveles (crecimiento económico y salud) pueden ser diferentes si el análisis se hace en términos de su distribución (asociación de varianzas), ya que los datos tienen que demostrar una historia diferente: países más iguales en salud (no importan sus niveles absolutos) parecen lograr una sociedad más inclusiva (menos desiguales en renta, no importa su nivel). $\mathrm{O}$ en términos inversos, las sociedades de renta menos desigual pueden lograr comunidades más iguales en salud. Pero, por supuesto, puede ser también el caso de que los países ricos (independientemente de su distribución interna de la renta) muestren preocupación, incluso quizás en aras de preservar el status quo, para evitar las desigualdades en salud. En resumen, los trabajos empíricos para poner a prueba aquellas afirmaciones adolecen de los problemas propios de causalidad inversa y sus estrategias de abordaje cambian si se analizan en términos de niveles o de variaciones. Por último, puede discutirse si las variables adecuadas para identificar aquellas asociaciones entre el desarrollo económico y la salud son las tasas absolutas (i) el PIB, o (ii) en términos per cápita, y cuál es el mejor índice de salud asociados a ellos, ya sea la mortalidad, la esperanza de vida, la auto evaluación de la salud, años de vida ajustados por calidad, etc.

Sobre la cuestión específica de encontrar proxies empíricas para el crecimiento económico, la renta, la utilidad o bienestar residual y el patrimonio neto son los candidatos obvios para instrumentar sus respuestas. Los índices de desigualdad para cada una de estas variables con el fin de identificar sus vínculos con el crecimiento pueden abordarse ya sea por el coeficiente de Gini o por otras medidas de polarización, dado que las posiciones nucleares en los extremos de la distribución pueden ser más cruciales que las distribuciones normales. Para la medición de la salud, la mortalidad prenatal y la esperanza de vida son las variables habituales. Sin embargo, pueden ser muy dependientes de factores tales como el aborto y la fertilidad en general. Otras proxy tales como la carga de la enfermedad tratan de incorporar aquellos aspectos de la economía en la esperanza de vida, al igual que lo hace el índice de desarrollo humano (IDH) para otras cuestiones de bienestar social.

Respecto de los factores puramente exógenos o correlacionados con la salud y el crecimiento, el gasto sanitario puede jugar un papel intermedio importante, 
ya que un mayor PIB implica o bien una mayor disposición privada a gastar en el cuidado de la salud o por medio de mayores bases imponibles, puede suponer más ingresos fiscales y el gasto sanitario público. Y a pesar de que sabemos que no siempre y no en cualquier etapa de desarrollo de un país, más gasto significa una mejor salud, se trata de candidatos típicos para controlar la endogeneidad.

\section{LA IMPORTANCIA DE LA UTILIZACIÓN DE SERVICIOS EN LOS SISTEMAS UNIVERSALES DE SALUD PARA LA VALORACIÓN DE SUS IMPACTOS REDISTRIBUTIVOS}

Normalmente entendemos la universalidad de un servicio público en sus aspectos relativos a la facilitación de acceso al servicio independientemente de la capacidad económica de las personas. Y lo hacemos normalmente pensando en los que menos tienen, los más frágiles o necesitados desde el punto de vista sanitario. Sin embargo, el universalismo es simétrico: permite el acceso, libre de coste monetario, también para los grupos de renta alta. De manera que si la distribución de necesidad relativa fuera igual y la propensión a su tratamiento idéntica entre colectivos, el universalismo generaría un consumo proporcional al peso de la población en cada estrato analizado. Ciertamente el mundo real es más complejo y cabe debatir cómo implementar la universalidad cuando, como en el caso español, la distribución de necesidades relativas y consumos es desigual (Van Doorslaer et al., 2006) y la elección social es sensible a los resultados en equidad (Williams, 2001).

Las genéticas y evoluciones comentadas, entendidas bajo la tendencia general hacia el universalismo, han sufrido recientemente algunas afectaciones a raíz de las políticas que los distintos países han desarrollado, en mayor o menor medida, como respuesta a la crisis económica. De acuerdo con el informe Health policy responses to the financial crisis in Europe (Mladovsky et al., 2012), las políticas impulsadas en salud pasan por recortes en los presupuestos de salud, reducción de la cartera de servicios y de la cobertura poblacional, así como medidas orientadas a reducir los precios de la atención sanitaria (afectando salarios, regulación farmacéutica y potenciando la centralización organizativa).

En general, podemos afirmar que una sanidad universal es redistributiva sólo en la medida que los grupos de renta elevada renuncian voluntariamente a consumir servicios públicos y optan por los privados. Pero ¿por qué razón hacerlo si la sanidad pública es tan excelente y gratuita? ¿Por qué pagar seguro privado si ni siquiera se puede compensar la parte que el Estado se ahorra? Que sea universal una prestación puede tener otras virtudes (las clases medias participan de los servicios a los que así les conviene dar apoyo), pero sin duda al coste de perder el foco de la priorización. La priorización, como cualquier forma de ordenación, no 
suele ser apetecible para los políticos que así evitan discriminar. Tampoco a los gestores públicos hacer algo diferente a atender la demanda que emerge. Lástima que las políticas se mantengan desde las inercias sin demasiado análisis sobre la efectividad social, al amparo tan sólo de la búsqueda del voto, ya sea por el miedo a los cambios o por mantener las promesas de beneficios gratuitos.

Existe una extensa controversia sobre la cuestión de si la orientación de las transferencias sociales hacia la parte inferior de la distribución del ingreso en realidad mejora o debilita su impacto redistributivo. Korpi y Palme (1998) han afirmado en un influyente trabajo que "cuanto más dirigimos los beneficios a los pobres, menos probable es que tengamos éxito en reducir la pobreza y la desigualdad”. El fundamento empírico de esta afirmación es una fuerte relación inversa a nivel de país entre la orientación de transferencia y el impacto social redistributivo.

En su informe de 2011 sobre la desigualdad Divided We Stand, la OCDE insta a "políticas de apoyo a los ingresos bien enfocadas", aunque sin especificar claramente qué forma y fuerza esto debe tomar. Por la misma razón, la Comisión Europea (2013) ha puesto en marcha un "paquete de Inversión Social también pidiendo una mayor focalización y condicionalidad: el apoyo debe enfocarse mejor a los necesitados y en el momento que más lo necesitan”. Organizaciones como el FMI y el Banco Mundial han defendido durante mucho tiempo los beneficios específicos, concretamente en forma de redes de seguridad social con comprobación de medios. En el contexto de crecientes demandas sobre los sistemas de asistencia social, es probable que se convierta aún más relevante la demanda de una mayor focalización. En la misma corriente de opinión, Goodin y Le Grand (1987: 215) estiman que "la participación beneficiosa de los no pobres en el Estado del Bienestar no es simplemente un desperdicio -en realidad es contraproducente-. Cuanto más se benefician los pobres menos redistributivo será el impacto del Estado del Bienestar".

Los mayores costos administrativos, problemas de estigmatización y discontinuidades derivadas de las trampas de dependencia están en el lado oscuro de esas proposiciones. Sin embargo, una cuestión más sustancial es la cantidad de recursos que en realidad pueden estar disponibles para su redistribución en virtud de los sistemas de bienestar selectivos: si menos fondos son la respuesta, ello puede afectar en efecto la capacidad total de la redistribución.

El plantearse en cualquier caso la priorización en el gasto parece a todas luces necesario a la vista de la naturaleza redistributiva (regresiva) de los ingresos que financien dicho gasto. En situaciones de "dualidad fiscal" en las que gana peso la imposición indirecta (regresiva ciertamente ya que en proporción pagan más los pobres que los ricos, dada la dedicación de los primeros al consumo de prácticamente toda su renta) y que entre los impuestos directos las rentas de capital acaban siendo mejor tratadas fiscalmente que las del trabajo (por aquello de su 
más fácil deslocalización en el mercado global), no puede caber duda de que en los márgenes al menos hace falta recuperar por la vía del gasto la progresividad perdida por la de los ingresos.

En el ámbito empírico, como hemos visto, tenemos que decidir cómo se va a medir la redistribución, ya sea mediante la comparación de la desigualdad de los ingresos realmente observada o con respecto a las personas en riesgo de pobreza. Ello requiere algunas distribuciones más o menos sofisticadas, "de contraste" (por ejemplo en torno a quién obtiene qué después de transferencias sociales y antes de impuestos; quién obtiene qué antes de impuestos y transferencias; quién obtiene qué después de impuestos y transferencias...). En teoría, ello debería reflejar con precisión la distribución del ingreso hipotético que prevalecería en ausencia de transferencias sociales, pero esto no prueba ser fácil.

Por lo que sabemos, la mayoría de los estudios anteriores han demostrado que la atención primaria suele ser "pro pobre", y la especializada y hospitalaria en su mayoría "pro rico" (sobre todo cuando se ajusta por "necesidad" (ver para el caso de Suecia Agerholm et al., 2013, y en términos similares para España, Urbanos, Ayala, Rivera et al., 2011 y en el Reino Unido, Cookson et al., 2010).

\section{Breve referencia al caso del gasto sanitario público en España}

En general, los principales estudios aplicados a España sobre la incidencia del gasto social público por niveles de renta, referidos a la década 1980-1990, ponían de relieve que el impacto redistributivo del gasto público sobre el gasto de los hogares se habría incrementado en esa década en más de un 24\%, siendo las prestaciones "en especie" las principales responsables de esa mejora y, singularmente, la contribución del gasto sanitario que acompañó la creación del Sistema Nacional de Salud (Gimeno, 1993), en menor medida, el gasto en educación.

Calonge y Manresa en 1992 fueron los primeros en mostrar que la utilización observada mostraba una contribución escasa a la redistribución del gasto sanitario público. Esto significa que el consumo sanitario público entre los deciles de renta en términos absolutos varía muy poco. La financiación per cápita de los proveedores sin ningún tipo de ajuste por renta o riqueza, en el proceso de descentralización de los recursos también es una prueba de esta visión que se otorga a los servicios universalistas.

Calero y Gil (2014) encuentran que el gasto sanitario público a pesar de ser algo progresivo (en términos absolutos, con un índice de concentración de $-0,1048$ ) y redistributivo (esto es, cumple con los objetivos de equidad que a priori se atribuyen a la política sanitaria), sin embargo, sus índices de progresividad y redistribución en el 2010 son peores que los obtenidos en 2005, si bien su papel redistribuidor relativo es más importante, puesto que permiten reducir la desigualdad en 2010 en mayor medida que en el 2005. El gasto sanitario beneficia 
en gran medida a los grupos sociales más desfavorecidos y de forma considerable a las clases medias. Sin embargo, respecto a 2005, este gasto ya no supone un peso tan elevado en relación a su renta para el colectivo de hogares más pobres, mientras que para los hogares más ricos, este peso es superior. Concluyen que los mayores contribuyentes a la progresividad son los gastos en atención primaria, seguidos por los gastos en urgencias. Estos últimos presentan en el 2010 un comportamiento diferente al obtenido en 2005, desbancando la capacidad progresiva de los gastos farmacéuticos, que en 2010 es mucho menor.

En general, en cuanto a su evolución, se puede decir que tanto la situación de partida (renta inicial) como la de llegada (renta final) es peor en términos globales en 2010 que en 2005, en lo que atañe a la desigualdad de renta. El gasto público sanitario contribuye en gran medida a paliar este empeoramiento en la equidad, aunque si comparamos con las décadas previas, se puede concluir que se vuelve a los valores similares a los de la década de los noventa, tanto en términos de progresividad como de redistribución. Aunque para dichos autores, el mayor lapso de tiempo entre las fechas de ambos análisis (2005 frente a 2010) en el caso de sanidad, y el ligero cambio en uno de los puntos de la metodología de fusión estadística (uso de la variable educación en lugar de la variable intervalo de renta) pueden explicar la mayor magnitud del cambio de tendencia en el caso sanitario, por ejemplo frente a lo acontecido con el gasto educativo.

En un estudio relativo el periodo 1987-2001 (García y López, 2007) constataban sus autores que, a igual nivel de necesidad, las diferencias de renta no daban lugar en España a diferencias en el acceso a la asistencia sanitaria (consultas con médicos, visitas a urgencias y hospitalización).

Abásolo et al (2014) evidencian que no existe equidad en el acceso a los servicios del sistema sanitario público por niveles socioeconómicos. A esa conclusión se llega cuando se considera simultáneamente las dos vertientes del acceso al sistema sanitario público: utilización y tiempos de espera, metodología que parece adecuada una vez que se evidencia que los que utilizan los distintos servicios sanitarios analizados tienen características distintas a los que no lo utilizan (i.e. existe un problema de sesgo de selección). En concreto, concluyen estos autores en relación con los servicios de medicina especializada y hospitalaria, que los sesgos de información y de costes de oportunidad del consumo provocan que los individuos con menor nivel socioeconómico tengan mayores problemas de acceso, evidenciados no sólo por una menor probabilidad de utilización sino por una mayor espera ( $\sin$ trato a favor); diferencias que no explica la necesidad sanitaria y otros factores socio-demográficos. Por su parte, los resultados del acceso no son tan concluyentes respecto a los servicios de medicina general: mientras los individuos con menor nivel socioeconómico, medido tanto a través del nivel de estudios como de la renta, tienen una mayor probabilidad de acudir al médico general, los individuos con menor nivel educativo muestran mayores tiempos de espera. 
Para un período más reciente como el observado con la crisis cabe intuir que si el gasto sanitario privado es elástico al ingreso, un efecto esperable es que incida mayormente en los deciles de ingresos medio-bajos de la población. Los menores ingresos pueden hacer cambiar la utilización de la atención primaria, la parte más favorable a los pobres de la redistribución de la renta implícita en los servicios de atención sanitaria en especie, tal como hasta hoy se había observado. Una primera lectura de los datos apuntan a este factor. Esta ha disminuido en España entre 2006 y 2011 alrededor de 20\% y 30\%, en particular para los grupos de bajos ingresos, previsiblemente, por no poner en riesgo el empleo con el absentismo laboral (médicos de atención primaria firmando licencias), por el papel más importante otorgado a las enfermeras para pluripatologías y tratamientos fuera de los centros de atención primaria, amén de algunas innovaciones como la receta electrónica que suelen evitar algunas visitas. Sin embargo, de la lectura simple de los datos se puede observar que la atención hospitalaria (que es predominantemente 'pro ricos') muestra una frecuentación más permanente y en este sentido menos afectada por la crisis. Algunas preguntas adicionales que se derivan de la perspectiva anterior son del tipo: ¿qué efecto producen las reducciones marginales del gasto público sanitario -consecuencia de la crisis económica- por grupos de renta (cuartiles de renta)? ¿Se ven más perjudicados los cuartiles de renta inferiores porque los más ricos abandonan la doble cobertura sanitaria y se incorporan a un sistema sanitario con menos recursos? ("efecto desplazamiento forzado" de los pobres). ¿O se produce el efecto contrario: ante el deterioro de la sanidad pública, aumenta el número de individuos de los cuartiles de renta más altos con seguro privado, beneficiando relativamente más a los cuartiles inferiores de renta? ("efecto deslizamiento voluntario" de los ricos).

Nótese que para el objetivo anterior se trata de analizar el efecto redistributivo y por tanto la utilización sanitaria efectiva $-\mathrm{y}$ no sus probabilidades- por grupos de renta. Hasta ahora la mayor parte de la literatura ha considerado el efecto conjunto no sólo de la renta sino de otras variables (educación, ocupación, situación laboral, etc.) de manera que -aparte de los posibles problemas de multicolinealidad entre dichas variables- el efecto redistributivo (efecto renta) ha quedado oculto o se ha difuminado sin llegarse a medir propiamente. Para ello, hace falta controlar por distintos factores que asumimos que podrían influir en la utilización sanitaria, tales como la composición demográfica por grupos de renta, género y nivel educativo. Por lo demás conviene conocer si ha cambiado la distribución de la necesidad sanitaria de los grupos de renta (especialmente por la autopercepción del estado de salud (que puede ser sensible a otros aspectos del bienestar personal relacionados con la crisis económica) o por el padecimiento de enfermedades crónicas que, aunque auto-declaradas, puedan tener un diagnóstico clínico previo. 


\section{CONCLUSIONES Y ALGUNAS REFLEXIONES SOBRE LA POLÍTI. CA ECONÓMICA, SOCIAL Y SANITARIA}

De modo resumido se ofrece a continuación unos breves destacados y una reflexión un poco más general de cierre del análisis.

A modo de conclusión, del estudio efectuado se derivarían como medidas de política social general:

- La necesidad de prestar más atención de la investigación en Economía de la Salud a los efectos a largo plazo de la crisis, aún cuando esta esté coyunturalmente superada. La relación entre epidemiología y los determinantes macrosociales de la salud son sus instrumentos vehiculares.

- La crisis requiere en el corto plazo una mejor orientación de los tratamientos paliativos en favor de su población relativamente más necesitada: instrumentos más a medida, desde políticas coordinadas horizontalmente, atendiendo a sus finalidades en términos de beneficiarios- objetivo.

- Desde el punto de vista de eficiencia, debería de constituir una clara ventaja el invertir en los niños y en su educación: la parte débil de la cadena de valor de la salud y la que exhibe mejores tasas de rendimiento de la aplicación de recursos públicos.

- En favor de la equidad intergeneracional debería adoptarse una visión más dinámica de la política social, que incluya

a) una reforma más gradual en el ajuste del gasto social que contemple una nueva combinación de fuentes de financiación: contribuciones públicas, privadas y comunitarias reguladas;

b) un sistema de contabilidad horizontal que analice la situación de bienestar de los grupos de destinatarios específicos y franjas de edad resultantes de la administración de los distintos presupuestos verticales, con seguimiento de recursos y efectos alcanzados.

Las anteriores medidas podrían acompañarse con algunas reflexiones adicionales. La idea prevalente en la mayor parte de las investigaciones en Economía de la Salud en relación a las desigualdades en salud se centra en aquellas vinculables a la renta a sus factores sociales determinantes. Su asociación con la crisis económica vivida va desde los que hablan de "austericidio", por la vía intermedia de los recortes sociales a los que en el otro lado del atlántico han llegado a asociar crisis económica, paro y mejora de estilos de vida por mayor oportunidad de estilo de vida saludable y reducción forzada por pérdida de renta de la ingesta de alcohol y tabaco. 
En cualquier caso, la idea que aquí defendemos es que, para validar las hipótesis previsibles de incidencia de la crisis económica sobre la salud y sobre las desigualdades en salud en particular, cabe tener en cuenta lo siguiente:

1. Que transcurre el plazo de tiempo apropiado entre el antes, durante y después para que los métodos empíricos detecten la naturaleza de los cambios. Y con atención a aspectos tales como el retardo en efectos o el momento efectivo que la crisis percibida se internaliza efectivamente en el bolsillo de los afectados. Por ejemplo, la crisis económica que en España se inicia en el segundo semestre del 2007 sólo en el segundo del 2010 de traduce en una pérdida real de la renta familiar disponible a la vista de las prestaciones sociales y medidas compensatorias en vigor (Coveney et al., 2015).

2. Los análisis transversales difícilmente pueden tener en cuenta todas las variables que entre países o estados entran en consideración a la hora de validar los efectos. Aún no exento de dificultad, el enfoque temporal resulta más sencillo por acotable.

El tema de impactos en salud es diferente al de los efectos en desigualdades. El ranking de individuos a igual o mejor salud puede afectar a las desigualdades. Por ejemplo, con la crisis es evidente que los jóvenes se han llevado la peor parte, y no por pérdida de salud sino por pérdida de renta. Nuestros mayores suelen ser más iguales en renta (aunque no en riqueza como forma de renta acumulada) que nuestros jóvenes, pero para estos últimos los efectos de estas diferencias sobre la salud son previsiblemente menores para cada grupo de edad. Sea cual sea la diferencia de renta es difícil que ello se traduzca en grandes varianzas de salud (todos nuestros mayores están más o menos iguales de pachuchos a igual edad). Si es la renta o la riqueza el mayor determinante para la salud, su signo, su linealidad o concavidad o si influye la composición de los activos financieros dada la distinta exposición al riesgo del ciclo económico, es algo ciertamente discutido. La misma composición demográfica aquí puede ser decisiva con el envejecimiento relativo (mas mayores en cuantía y porcentaje que los jóvenes, lo que dimensiona el efecto antes comentado) o en razón de la entrada de inmigración en la coyuntura económica favorable y emigración con la crisis. Es además dudoso que el efecto sobre las desigualdades detectadas por los índices de concentración al uso debido al re-ordenación se mantenga en salud si los grupos etarios son substituidos por otras clasificaciones tales como familias monoparentales, hogares con dos perceptores de renta, individuos adultos no emancipados en unidades familiares compuestas... y obviamente según educación y/o ocupación.

La medición tomada como salud puede igualmente marcar los resultados. Notemos que si utilizamos salud autopercibida los que se quedan en el camino no acaban contando, a diferencia de si tomamos la mortalidad, sensible a las tasas 
de suicidio pero no tanto en los accesos ordinarios a los servicios de salud. Por supuesto como otras cosas importan además de la salud uno podría alegar que indicadores de satisfacción con la propia vida pueden aquí ser relevantes (permitiendo incluso una mayor cardinalidad que los estratos de niveles de salud o el binomial vida/ muerte). Y acorde con ello, puede variar en el tiempo la propia relación entre renta y salud (auto-valorada, satisfacción, enfermedad o muerte), en sus mecanismos de transmisión y en sus interacciones precisas, más o menos aminoradas por políticas sociales variantes en el tiempo.

Finalmente cabe tomar seriamente la evaluación del riesgo de introducción de sesgos en el análisis anterior. De modo que serían sesgos del análisis?

(I) El sesgo de selección de la muestra no es representativa de la población destinada a ser analizada, no siendo probable que las observaciones seleccionadas para participar en el estudio sean representativas de la población objetivo. Por ejemplo, para los estudios de la encuesta, una tasa de respuesta de $>80 \%$ es de bajo riesgo, $60-79 \%$ es riesgo moderado y $<60 \%$ o la tasa de respuesta no se informa es de alto riesgo;

(II) falacia ecológica: falacia lógica inherente en la consideración de causalidad desde inferencias a partir de los datos del grupo a individuo (por ejemplo en comportamientos);

(III) sesgo de confusión: el análisis no tiene en cuenta los factores de confusión que se espera tengan un efecto en el resultado y que no han sido explicados ya por la inclusión de otra variable;

(IV) sesgo de información: por información inapropiada o imprecisa de los aspectos del estudio. Por ejemplo, son todos los aspectos del estudio, incluyendo objetivos, métodos y resultados, descritos con claridad y reportados correctamente;

(V) sesgo temporal: sesgo introducido debido a los plazos considerados. Fueron los análisis realizados de forma adecuada teniendo en cuenta períodos de más de 10 años de datos, al menos tres años de posterior a la crisis y considerando en su caso los posibles efectos del retraso en los efectos;

(VI) de instrumentos de medición: los errores relacionados con la variable de exposición se producen al considerar la crisis económica con variables explicativas macroeconómicas de más del año de la crisis, y

(VII) sesgo de error de medición en el resultado de salud, ya desde un indicador clínico o de datos registrados oficialmente que no es probable que sean misreported o inexactos hasta de resultados auto declarados, con o

${ }^{7}$ Adaptado de Parmar, Stavropoulou y Ioannidis. (2016). De todos los trabajos analizados sólo el de Zapata Moya, Buffel, Navarro Yáñez y Bracke (2015) y el de Gili, Roca, Basu, McKee y Stuckler (2013) consiguen una puntuación intermedia aunque no claramente no sesgada en muchos de los aspectos que se mencionan. 
sin herramienta validada, mecánicamente o por funcionario; así para el registro de datos que son propensos a inexactitudes (por ejemplo, el suicidio en el certificado de defunción). Todo ello clama para que las conclusiones de estudios, análisis, asociaciones y causalidades pretendidas mantengan elevadas dosis de precaución.

\section{BIBLIOGRAFÍA}

ABÁSOLO, I., NEGRÍN-HERNÁNDEZ, M. y PINILLA J. (2013), "Utilización y tiempos de espera: dos vertientes inseparables del análisis de la equidad en el acceso al sistema sanitario público", Hacienda Pública Española, núm. 208, pp. 11-38.

AGERHOLM, J. et al. (2013), "Socioeconomic differences in healthcare utilization, with and without adjustment for need: an example from Stockholm, Sweden", Scandinavian Journal of Public Health, vol. 41, núm. 3, pp. 318-325.

BHARGAVA, A. et al. (2001), "Modeling the effects of health on economic growth", Journal of Health Economics, vol. 20, núm. 3, pp. 423-440.

CALONGE, S. y MANRESA, A. (1997), "Consecuencias redistributivas del Estado del Bienestar en España: Un análisis empírico desagregado", Moneda y crédito, núm. 204, pp. 13-65.

CALERO, J. y GIL, M. (2014), Un análisis de la incidencia distributiva del gasto público en sanidad y educación en España. Informe sobre exclusión y desarrollo social en España, Fundación Foessa, Madrid.

JAGGER, C. et al. (2008), "Inequalities in healthy life years in the 25 Countries of the EU in 2005", The Lancet, núm. 372, pp. 2124-2131.

CONTOYANNIS, P. y FORSTER, M. (1999), "The distribution of health and income: a theoretical framework", Journal of Health Economics, vol.18, núm. 5, pp. 605-622.

COOKSON, R., DUSHEIKO, M., HARDMAN, G. y MARTIN, S. (2010), "Competition and inequality: Evidence from the English National Health Service 1991-2001", Journal of Public Administration Research and Theory, núm. 20, pp. 181-205.

COVENEY, M. et al. (2015), "Health disparities by income in Spain before and after the economic crisis", Discussion Paper, Tinbergen Institute.

DEATON, A. (2013), The Great Escape: Health, Wealth and the Origins of Inequality, Princeton University Press, Princeton.

DEATON, A. y PAXSON, C. (1998), "Aging and inequality and health", Journal of Public Economics, núm. 102, pp. 437-467.

DOW, W. y SCHOENI, R. (2008), Economic Value of Improving the Health of Disadvantaged Americans, Technical Report for Overcoming Obstacles to 
Health: Report from the Robert Wood Johnson Foundation to the Commission to Build a Healthier America.

FLEURBAEY, M. y SCHOKKAERT, E. (2009), "Unfair inequalities in health and healthcare", Journal of Health Economics, núm. 28, pp. 73-90.

GARCÍA, P. y LÓPEZ, A. (2007), The evolution of inequity in the access to health care in Spain: 1987-2001, Fundación BBVA, Bilbao.

GILI, M., ROCA, M., BASU, S., MCKEE, M. y STUCKLER, D. (2013), "The mental health risks of economic crisis in Spain: evidence from primary care centres, 2006 and 2010", European Journal of Public Health, núm. 23, pp. 103-108.

GIMENO, J. (1993), "La incidencia del Gasto Público por niveles de renta: España 1990 vs. 1980", Ponencia presentada en el I Simposio sobre igualdad y distribución de la renta y la riqueza. Fundación Argentaria, vol. 7, pp. 63-121.

GOODINN, R. E. y LE GRAND, J. (1987), Not only the poor: the middle classes and the welfare state, Allen \& Unwin, Londres.

KNOWLES, S. y OWEN, P. D. (1995), "Health capital and cross-country variation in income per capita in the Mankiw-Romer-Weil model", Economic Letters, núm. 48, pp. 99-106.

KORPI, W. y PALME, J. (1998), "The Paradox of Redistribution and Strategies of Equality: Welfare State Institutions, Inequality, and Poverty in the Western Countries", American Sociological Review, vol. 63, núm. 5, pp. 661-687.

KUNST, A. y MACKENBACH, J. P. (1994), "The size of mortality differences associated with educational level in nine industrialized countries", American Journal of Public Health, núm. 84, pp. 932-937.

LEIGH, A., JENCKS, C. y SMEEDING, T. M. (2009), The Oxford Handbook of Economic Inequality, Oxford University Press, Oxford.

LÓPEZ-CASASNOVAS, G., CURRAIS, L. y RIVERA, B. (eds.) (2005), Health and Economic Growth, MIT Press, Cambridge.

LÓPEZ-CASASNOVAS, G. (2012), "Crisis económica, regresión de renta, desigualdades en salud", Revista de Gestión Clínica y Sanitaria, vol. 14, núm. 2-3, pp. 43-49.

LÓPEZ-CASASNOVAS, G. y SOLEY-BORI, M. (2014), "The Socioeconomic Determinants of Health: Economic Growth and Health in the OECD Countries during the Last Three Decades", International Journal of Environmental Research and Public Health, vol. 11, núm. 1, pp. 815-829.

LUNDBERG, O. et al. (2008), "The role of the principles of the welfare state and the generosity of social programs for Public Health", The Lancet, vol. 372, 8 de noviembre, pp. 1633-1640.

MACKENBACH, J. y STRONKS, K. (2002), "A strategy for tackling health inequalities in the Netherlands", British Medical Journal (BMJ), núm. 325, p. 7371. 
MACKENBACH, J. et al. (2011), "Economic costs of Health inequalities in the European Union", Journal of Epidemiology and Community Health, núm. 65, pp. 12-19.

MAZZUCO, S., MEGGIOLARO, S. y SUHRCKE, M. (2010), "The economic benefits of reducing health inequalities in England and Wales". (Background Report to the Marmot Review).

MLADOVSKY, P. et al. (2012), "Health policy in the financial crisis in Europe", Eurohealth, vol. 18, núm.1.

OECD (2009), Measuring Disparities in Health Status and Access.

PARMAR, D., STAVROPOULOU, C. y IOANNIDIS J. P. (2016), "Health outcomes during the 2008 financial crisis in Europe: systematic literature review", British Medical Journal (BMJ), núm. 354, p. 4588.

RODRÍGUEZ, M. y GONZÁLEZ LÓPEZ-VALCÁRCEL, B. (2011), "Alas, there are no shortcuts to the complexities of the economy", Journal of Epidemiol Community Health, vol. 65, p. 389.

ROEMER, J. (1996), Theories of Distributive Justice, Harvard University Press, Harvard.

ROEMER, J. (1998), Equality of Opportunity, Harvard University Press, Harvard.

SUHRCKE, M. y URBAN, D. (2010), “Are cardiovascular diseases bad for economic growth?", Health Economics, vol. 12, núm. 19, pp. 1478-1496.

SUHRCKE, M. y COOKSON, R. (2012), Using Economic Evidence to Support the Case for Action to Tackle Health Inequality, Economics Task Group, Londres.

TODESCHINI, F. (2010), Essay on the health and labor consequences of unhealthy habits, Tesis doctoral, Universitat Pompeu Fabra.

URBANOS, R. (2001), "Explaining Inequality in the Use of Public Health Care Services: Evidence from Spain”, Health Care Management Science, núm. 4, pp. 143-157.

VAN DOORSLAER, E., MASSERIA, C. y KOOLMAN, X. (2006), "Inequalities in Access to Medical Care by Income in Developed Countries", Canadian Medical Association Journal, vol. 174, núm. 2, pp.177-183.

VAN OURTI, T., VAN DOORSLAER, E. y KOOLMAN X. (2009), "The effect of income growth and inequality on Health inequality: theory and empirical evidence from the European Panel", Journal of Health Economics, núm. 28, pp. 525-539.

WILLIAMS, D. R. (2001), "Racial residential segregation: a fundamental cause of racial disparities in health", Public Health Report, vol. 116, núm. 5, pp. 404-416.

ZAPATA MOYA, A. R., BUFFEL, V., NAVARRO YÁÑEZ, C. J. y BRACKE, P. (2015), "Social inequality in morbidity, framed within the current economic crisis in Spain", International Journal of Equity Health, núm. 14:131, pp. 1-20. 